\title{
Influence of mesenchymal stem cell- derived extracellular vesicles in vitro and their role in ageing
}

Juan Fafián-Labora ${ }^{1,2+}$, Miriam Morente-López ${ }^{1,2+}$, María José Sánchez-Dopico ${ }^{1,2}$, Onno J. Arntz ${ }^{3}$, Fons A. J. van de Loo ${ }^{3}$, Javier De Toro ${ }^{1,2}$ and María C. Arufe ${ }^{1,2^{*}}$ (D)

\begin{abstract}
Introduction: This study assessed whether mesenchymal stem cell (MSC)-derived extracellular vesicles influenced ageing and pluripotency markers in cell cultures where they are added.

Methods: MSC-derived extracellular vesicles from old and young rat bone marrows were isolated by ultracentrifugation and were characterised by western blotting, nanoparticle tracking analysis (NTA) and transmission electron microscopy (TEM). They were added to young and old MSC cultures. Real-time quantitative reverse transcription polymerase chain reactions and western blot analysis were performed to check the markers of ageing (vinculin and lamin A), pluripotency markers (Nanog and Oct4) and components of the mTOR signalling pathway (Rictor, Raptor, AKT and mTOR) in these cell populations. Subsequently, microRNA (miR)-188-3p expression was transiently inhibited in young MSCs to demonstrate the influence of mTOR2 on MSC ageing.
\end{abstract}

Results: Incubation with young MSC-derived extracellular vesicles decreased the levels of ageing markers and components of the mTOR pathway and increased the pluripotency markers from old MSC populations. By contrast, incubation of young MSCs with old MSC-derived extracellular vesicles generated the reverse effects. Inhibition of miR-188-3p expression in young MSCs produced extracellular vesicles that when incubated with old MSCs produced an increase in the levels of Rictor, as well as a decrease of phosphor-AKT, as indicated by a significant decrease in beta-galactosidase staining.

Conclusions: MSC-derived extracellular vesicles affected the behaviour of MSC cultures, based on their composition, which could be modified in vitro. These experiments represented the basis for the development of new therapies against ageing-associated diseases using MSC-derived extracellular vesicles.

Keywords: Mesenchymal stem cell-derived extracellular vesicles, Ageing, Pluripotency, mTOR pathway

\section{Introduction}

Cellular senescence, the result of complex phenotypic changes and ageing, constitutes a great risk factor that contributes to trigger different degenerative diseases. Unravelling the importance of the effect of ageing on mesenchymal

\footnotetext{
* Correspondence: maria.arufe@udc.es

†Juan Fafián-Labora and Miriam Morente-López contributed equally to this work.

'Grupo de Terapia Celular y Medicina Regenerativa, Instituto de Investigación Biomédica de A Coruña (INIBIC), Complexo Hospitalario Universitario de A Coruña (CHUAC), 15006-A Coruña, Spain

${ }^{2}$ Departamento de Fisioterapia, Ciencias Biomédicas y Medicina, Facultad de Ciencias de la Salud, Universidad de A Coruña (UDC), As Xubias, 15006-A Coruña, Spain

Full list of author information is available at the end of the article
}

stem cells (MSCs) is crucial in the development of new therapy based on these cells, focusing on the treatment of older people. The beneficial effects on damaged tissues are attributed to the paracrine activity of the MSCs, which include their soluble factors and extracellular vesicles (EVs). An interesting study indicated that MSC-derived EVs have significant potential as a novel alternative to whole-cell therapies in an experimental preclinical model of the inflammatory lung [1]. EVs transport mRNA, microRNA (miRNA) and proteins giving them the possibility of transporting biological information to the surrounding cells [2]. Previous results by our group indicated that proliferative, pluripotency and metabolism profiles of MSCs have

(c) The Author(s). 2019 Open Access This article is distributed under the terms of the Creative Commons Attribution 4.0 International License (http://creativecommons.org/licenses/by/4.0/), which permits unrestricted use, distribution, and 
significant differences influenced by the donor age [3]. We also demonstrated that immune profiles of MSC-derived EVs have age-dependent differences and they can be modified using miRNA [4]. The mammalian target of rapamycin (mTOR) is an amino acid sensor which perceives and incorporates different environmental signals. mTOR balances cell growth and nutrient availability and contributes to decisions on stem cell fate as a consequence of endogenous DNA damage [4]. mTOR also regulates cellular senescence and drives the infrastructure of bioenergetics [5]. Rapamycin inhibits the senescence effect produced by mTOR, which is mediating MSC proliferation potential through its effect on their self-renewal loss [6]. There are two signalling complexes, mTOR complex 1 (mTORC1) and mTOR complex 2 (mTORC2). mTORC2 plays an important role in endothelial senescence, evident by an increase in binding of Rictor, which is an essential component of mTORC2directed phosphorylation of mTOR at Ser2481 and AKT (phosphor-AKT), producing an increase of $\beta$-galactosidase staining in senescence [7]. MicroRNA (miR)-188-3p has been shown to be a key regulator of age-associated bone loss in the bone marrow through its target on Rictor [8], and insights into the mechanism involved in MSC ageing suggest that interventions with miRNAs could modify the function of MSCs and their derived extracellular vesicles [4]. This study assessed whether EVs could modify MSC markers of ageing in vitro and whether Rictor has a key role through the mTORC2 pathway involving the ageing process.

\section{Material and methods} Isolation and culture of MSCs

Animals were anesthetised with Fluorane (Izasa, A Coruña, Spain) and euthanised by the cervical dislocation method. The femoral bones were dissected from male Wistar rats (Animal Service, CHUAC, Spain) at different ages, young (14 days old) and old (270 days old). All methods were carried out following the approved guidelines of the Spanish Law (32/2007). All experimental protocols were approved by the Animal Ethical Committee of Galicia. The protocol published by Karaoz et al. [1] was used in this work. Briefly, the ends of the femoral bones were cut away, and a 21-gauge needle inserted into the shaft of the bone marrow was extruded by flushing with $4 \mathrm{ml} \mathrm{D-Hank's} \mathrm{solution} \mathrm{supplemented}$ with $100 \mathrm{IU} / \mathrm{ml}$ penicillin- $100 \mathrm{mg} / \mathrm{ml}$ streptomycin (Life Technologies, Madrid, Spain). The marrow plug suspension was dispersed by pipetting and filtered through a $70-\mu \mathrm{m}$ mesh nylon filter (BD Biosciences, Bedford, MA, USA), successively centrifuged at $2000 \mathrm{~g}$ for $10 \mathrm{~min}$. The supernatant containing haematopoietic cells was discarded, and the cell pellet was resuspended in Roswell Park Memorial Institute (RPMI) medium supplemented with $10 \%(\mathrm{v} / \mathrm{v})$ foetal bovine serum (FBS), 1\% (v/v) penicillin and $1 \%(\mathrm{v} / \mathrm{v})$ streptomycin (Life Technologies, Madrid, Spain). The MSCs were plated into $100-\mathrm{cm}^{2}$ dish plates (Corning Inc., NY, USA) and incubated at $37^{\circ} \mathrm{C}$ in a humidified atmosphere of $5 \% \mathrm{CO}_{2}$. MSCs were isolated because of their ability to adhere to the culture plates. On the third day, red blood cells and other non-adherent cells were removed by a pre-plating technique, and fresh medium was added to allow further growth to the MSCs. The adherent MSCs grown to 70\% confluence were defined as passage 0 (P0) cells. The culture medium was replaced every 3 or 4 days. MSCs were expanded for two passages before being used in the experiments. In 6-well plates (Corning Inc., NY, USA), $2.5 \times 10^{5}$ MSCs from old individuals were cultured per well for $8 \mathrm{~h}$, and $2 \times 10^{7}$ particles of MSC-derived EVs from young individuals were added to these wells, and vice versa. MSCs were collected after 2, 3 and 6 days in culture with different MSC-derived EVs, and RNA and protein isolations were performed. Young MSCs were incubated with $40 \mathrm{nM}$ miR-188-3p miRVAna ${ }^{\mathrm{Tm}}$ inhibitor or $40 \mathrm{nM}$ control negative miRVAna ${ }^{\text {TM }}$ Mimic using the expression system and protocols of the manufacturer. Validation by reverse transcription polymerase chain reaction (RT-PCR) was done using TaqMan ${ }^{\circ}$ MicroRNA Assays following the instructions of the manufacturer (Ambion, Applied Biosystems, Madrid, Spain). MSC cultures without added MSC-derived EVs were used as a control in all the experiments.

\section{Flow cytometry}

To characterise the MSCs, they were washed twice in phosphate-buffered saline (PBS; Sigma-Aldrich, St. Louis, MO, USA) then pre-blocked with $2 \%$ rat serum in PBS. The following direct antibodies were used: phycoerythrin (PE)-conjugated mouse anti-human CD34 (1:20; DakoCytomation, Barcelona, Spain), fluorescein isothiocyanate (FITC)-conjugated mouse anti-rat CD45 (1:20; BD Pharmingen, Franklin Lakes, NJ, USA), PE-cyanine (Cy)5.5conjugated mouse anti-rat CD90 (1:20; Immunostep, Salamanca, Spain) and allophycocyanin (APC)-conjugated mouse anti-rat CD29 (1:20; Immunostep, Salamanca, Spain). The cells were washed with PBS after $1 \mathrm{~h}$ of incubation with the corresponding antibody at room temperature. Fluorescence-activated cell sorting (FACS) data was generated by BD FACSDiva software (BD Science, San Jose, CA, USA). Negative control staining was performed using FITC-conjugated mouse IgG1K isotype, PE-conjugated mouse IgG1K isotype, PE-Cy5.5-conjugated mouse IgG1K isotype and APC-conjugated mouse IgG1K isotype (BD Pharmingen, Franklin Lakes, NJ, USA).

\section{Isolation of MSC-derived EVs}

MSCs from young (14 days) and old (270 days) rats were cultured with RPMI 1640 medium with 
GlutaMAX $^{\mathrm{m}}$ supplement, 10\% exosome-depleted FBS (Thermo Fisher Scientific, Waltham, MA, USA) and $100 \mathrm{IU} / \mathrm{ml}$ penicillin-100 $\mathrm{mg} / \mathrm{ml}$ streptomycin (Life Technologies, Madrid, Spain). Cells were cultured to $80 \%$ confluence, and the supernatants were collected after $48 \mathrm{~h}$. Supernatants were centrifuged at $2000 \times g$ for $10 \mathrm{~min}$ at $4{ }^{\circ} \mathrm{C}$ and filtered using a sterile $0.22-\mu \mathrm{m}$ filter (GE Healthcare Life Sciences, Little Chalfont, UK) to eliminate debris, and they were transferred into new ultracentrifugation tubes (Beckman Coulter, Mississauga, Canada) and centrifuged at $100,000 \times g$ for $2 \mathrm{~h}$ at $4{ }^{\circ} \mathrm{C}$ in an Optimal-90K ultracentrifuge with a $60 \mathrm{Ti}$ rotor (Beckman Coulter, Mississauga, Canada). The last supernatants containing exosome-depleted FBS were removed, and the pellets were resuspended in $200 \mu \mathrm{l}$ PBS (MP Biomedicals, Illkrich-Graffenstaden, France).

\section{Nanoparticle tracking analysis of MSC-derived EVs}

The Brownian motion of the particles in a NanoSight LM12 using Nanoparticle Tracking Analysis 2.3 software (NanoSight Ltd., Amesbury, UK) was used to calculate the EV size distribution after the ultracentrifugation. Total protein concentrations in MSC-derived EVs were determined with a Micro-bicinchoninic acid (BCA) kit (Thermo Fisher Scientific, Rockford, IL, USA), according to the manufacturer's instructions.

\section{Electron microscopy}

MSC-derived EVs were concentrated using Vivaspin concentrators (Sartorius, Gottingen, Germany). They were gathered up in small volumes of deionised water, which were then placed on nickel grids and allowed to dry for $45 \mathrm{~min}$ at $37^{\circ} \mathrm{C}$. The grids with MSC-derived EVs were fixed with $4 \%(\mathrm{w} / \mathrm{v})$ paraformaldehyde (Sigma-Aldrich, St. Louis, MO, USA) for $10 \mathrm{~min}$ and then washed by dipping them onto several drops of deionised water. The grids were examined on a Jeol JEM1400 transmission electron microscope (Jeol Ltd., Tokyo, Japan).

\section{Fluorescence microscopy}

MSCs $\left(2.5 \times 10^{5}\right)$ from old and young individuals were cultured on slides (Sigma-Aldrich, St. Louis, MO, USA) pre-treated with poly-D-lysine (Sigma-Aldrich, St. Louis, MO, USA) in 6-well plates (Corning Inc., New York, NY, USA) for $8 \mathrm{~h}$ (day 1). MSC-derived EVs $\left(2 \times 10^{7}\right)$ from young and old individuals were stained with $10 \mu \mathrm{M}$ 3-3'-diethylthiacarbocyanineiodide (DiI) and added to each culture of MSCs; PBS (MP Biomedicals, IllkrichGraffenstaden, France) was added as a control. At 2, 3 and 6 days, the cells were washed three times with PBS (MP Biomedicals, Illkrich-Graffenstaden, France) and fixed with $4 \%(\mathrm{w} / \mathrm{v})$ paraformaldehyde (Sigma-Aldrich, St. Louis, MO, USA) for $10 \mathrm{~min}$, and then the slides were mounted using ProLong ${ }^{\oplus}$ Gold antifade mountant with 4',6-diamidino-2-phenylindole (DAPI; (Thermo Fisher Scientific, Carlsbad, CA, USA). The cells were examined with an Olympus BX61 microscope using a DP71 digital chamber (Olympus, Tokyo, Japan) with software DP-Controller and DP-Manager software. A NeoScope JCM-6000 Plus scanning electron microscope (Nikon-Izasa, Barcelona, Spain) was used to take supplementary images.

\section{Reverse transcription quantitative PCR analysis}

Total RNA from culture cells was isolated with TRIzol $^{\circ}$ reagent (Thermo Fisher Scientific, Waltham, MA, USA). For miRNA detection, cDNA was generated from DNaseItreated RNA, using a QuantiMir RT Kit (System Biosciences, Palo Alto, CA, USA), according to the manufacturer's instructions. PCR products were amplified using specific primers for miRNAs, mmu-miR-188-3p and U6 small nuclear RNA (Thermo Fisher Scientific, Waltham, MA, USA). The amplification programme consisted of an initial denaturation at $50^{\circ} \mathrm{C}$ for $2 \mathrm{~min}$, followed by $95^{\circ} \mathrm{C}$ for $10 \mathrm{~min}$, and 50 cycles of annealing at $95^{\circ} \mathrm{C}$ for $15 \mathrm{~s}$ and extension at $60^{\circ} \mathrm{C}$ for $1 \mathrm{~min}$. Primers for the amplification of rat genes are described in Table 1. The amplification programme consisted of an initial denaturation at $92^{\circ} \mathrm{C}$

Table 1 Specific primers for real-time reverse transcriptase polymerase chain reaction (RT-PCR) amplification, listed with their annealing temperature (AT)

\begin{tabular}{llll}
\hline Gene name & Fw primer & Rv primer & mRNA ID \\
\hline Nanog & atgcctcacacggagactgt & aagtgggttgtttgcctttg & NM_005103.4 $\left({ }^{\circ} \mathrm{C}\right)$ \\
Oct4 & ctcctggagggccaggaatc & atatacacaggccgatgtgg & NM_00510 \\
Vinculin & aggagaccttgcgaagacagg & gcggttgccacttgtttag & NM_001107248 \\
LMNA & gagcaaagtgcgtgaggagt & tccccctccttcttggtatt & NM_001002016.2 \\
mTOR & atcaagcaagcgacatctca & caggccttggttaccagaaa & NM_019906.1 \\
AKT & gacgtagccattgtgaaggag & ccatcattcttgaggaggaagt & NM_033230.2 \\
Rictor & gttggaaaaatggcacaagg & ctgtatgtagtgagggcttcgtt & NC_005101.4 \\
HPRT & agccgaccggttctgtcat & agccgaccggttctgtca & NM_012583.2 \\
\hline
\end{tabular}

Fw forward, $R v$ reverse 
for $2 \mathrm{~min}$, followed by 40 cycles of annealing at $95^{\circ} \mathrm{C}$ for $15 \mathrm{~s}$; annealing at $55-62^{\circ} \mathrm{C}$, depending on the gene, for 30 s; and extension at $72{ }^{\circ} \mathrm{C}$ for $15 \mathrm{~s}$. PCRs were done in triplicate, with each set of assays repeated three times. To minimise the effects of unequal quantities of starting RNA and to eliminate potential sources of inconsistency, relative expression levels of each gene were normalised to ribosomal protein (HPTR) or U6 small nuclear RNA using the $2-\Delta \Delta C T$ method $x[9]$. Control experiments utilised no reverse transcriptase.

\section{Immunoblot analysis}

Immunoblot analysis was performed with $40 \mu \mathrm{g}$ total protein extracted from MSCs or $20 \mu \mathrm{g}$ total protein extracted from MSC-derived EVs. Proteins were separated according to their molecular weight using sodium dodecyl sulphate-polyacrylamide gel electrophoresis (SDS-PAGE), with the percentage $(\mathrm{w} / \mathrm{v})$ bis-acrylamide (Sigma-Aldrich, St. Louis, USA) of the resolving gels being determined by the size of the proteins. Proteins were then transferred to nitrocellulose membranes using a semi-dry method, using buffer with 20\% (v/v) methanol (Panreac, Barcelona, Spain) for small proteins $(<100 \mathrm{kDa})$ or $10 \%(\mathrm{v} / \mathrm{v})$ methanol (Panreac, Barcelona, Spain) for large proteins $(>100$ $\mathrm{kDa}$ ). Nitrocellulose membranes were then incubated for $1 \mathrm{~h}$ with agitation at room temperature in blocking buffer, consisting of $5 \%(\mathrm{w} / \mathrm{v})$ bovine serum albumin (BSA) for phospho-proteins and 5\% (w/v) milk (Sigma-Aldrich, St. Louis, USA). The membranes were probed with antibodies diluted in blocking buffer at $4{ }^{\circ} \mathrm{C}$ overnight. The following day, the membranes were washed three times for 5 min with Tris-buffered saline with $0.1 \%(\mathrm{v} / \mathrm{v})$ Tween $^{\circ}$ 20 (TBST). The membranes were then incubated for $1 \mathrm{~h}$ at room temperature in horseradish peroxidase (HRP)conjugated secondary antibodies diluted in blocking buffer. Next, the membranes were washed three times in TBST buffer for 5 min with agitation and twice using Tris-buffered saline (TBS) for 5 min with agitation. An
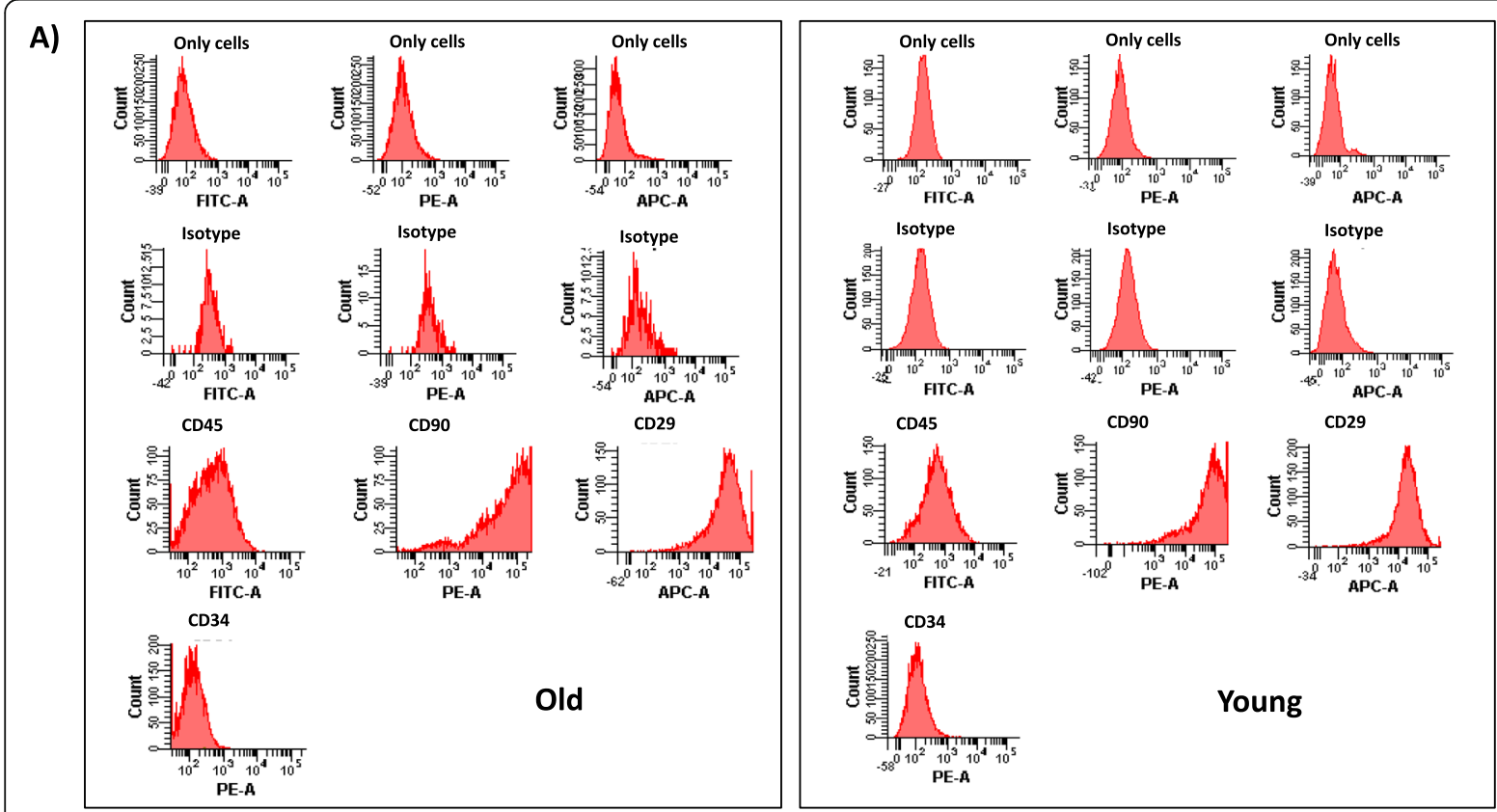

B)
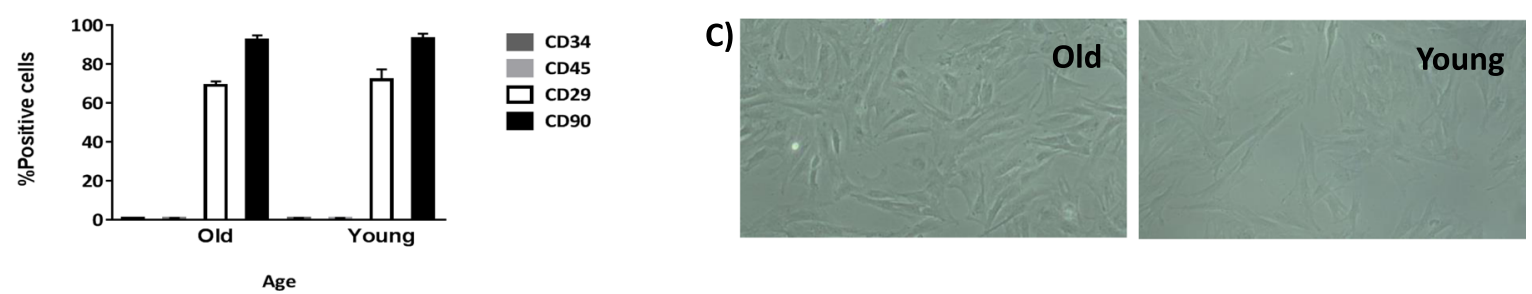

Fig. 1 Characterisation of MSCs from the rat bone marrow at several ages. a One representative fluorescence-activated cell sorting (FACS) assay is shown for old MSCs and young MSCs. The antibody is indicated at the top of each plot and its linked fluorochrome at the bottom. $\mathbf{b}$ The plot shows the percentage of positive MSC markers (CD29 and CD90) and negative haematopoietic markers (CD34 and CD45). c Representative images (magnification, $\times 20$ ) of old and young MSCS 
Amersham ECL Western Blotting Analysis System (GE Healthcare, Little Chalfont, UK) was used to visualise protein-binding antibodies. The blots were probed with antibodies directed against LMNA/C (Acrix); mTOR, Rictor, phosphor-mTOR, AKT and phosphor-AKT (Cell Signaling Technology, Beverly, MA, USA); Tsg101, calnexin and CD63 (Abcam, Cambridge, MA, USA); and $\beta$-actin (Sigma-Aldrich, St. Louis, MO, USA). Adequate concentrations for each antibody were determined empirically. Blot images were digitised using a LAS 3000 image analyser (GE Healthcare, Little Chalfont, UK). Densitometry analysis of band intensities was performed using ImageQuant 5.2 software (GE Healthcare, Little Chalfont, UK).

\section{Senescence-associated beta-galactosidase staining}

Cytochemical staining of senescence-associated betagalactosidase (SA- $\beta$-gal) with 5-bromo-4-chloro-3-indolyl$\beta$-D-galactopyranoside (X-Gal) was performed as previously described [7]. Cells were fixed and then stained with freshly prepared SA- $\beta$-gal staining solution overnight at $37^{\circ} \mathrm{C}$, according to the manufacturer's protocols (Cell Signaling Technology, Beverly, MA, USA). The experiments were conducted three times, with ten images being acquired of each treatment using an Olympus BX61 microscope (Olympus, Tokyo, Japan). Images were quantified with ImageJ software, with the intensity of SA- $\beta$-gal staining being expressed as arbitrary units.

\section{Statistical analysis}

All experiments were performed in triplicate, and one representative was shown in this paper. Non-parametric statistical analysis was performed by Mann-Whitney $U$ and Kruskal-Wallis tests using GraphPad Prism 6 (GraphPad Software, La Jolla, USA). $P$ values $<0.05$ were considered statistically significant. All the results were presented as standard errors of the mean.

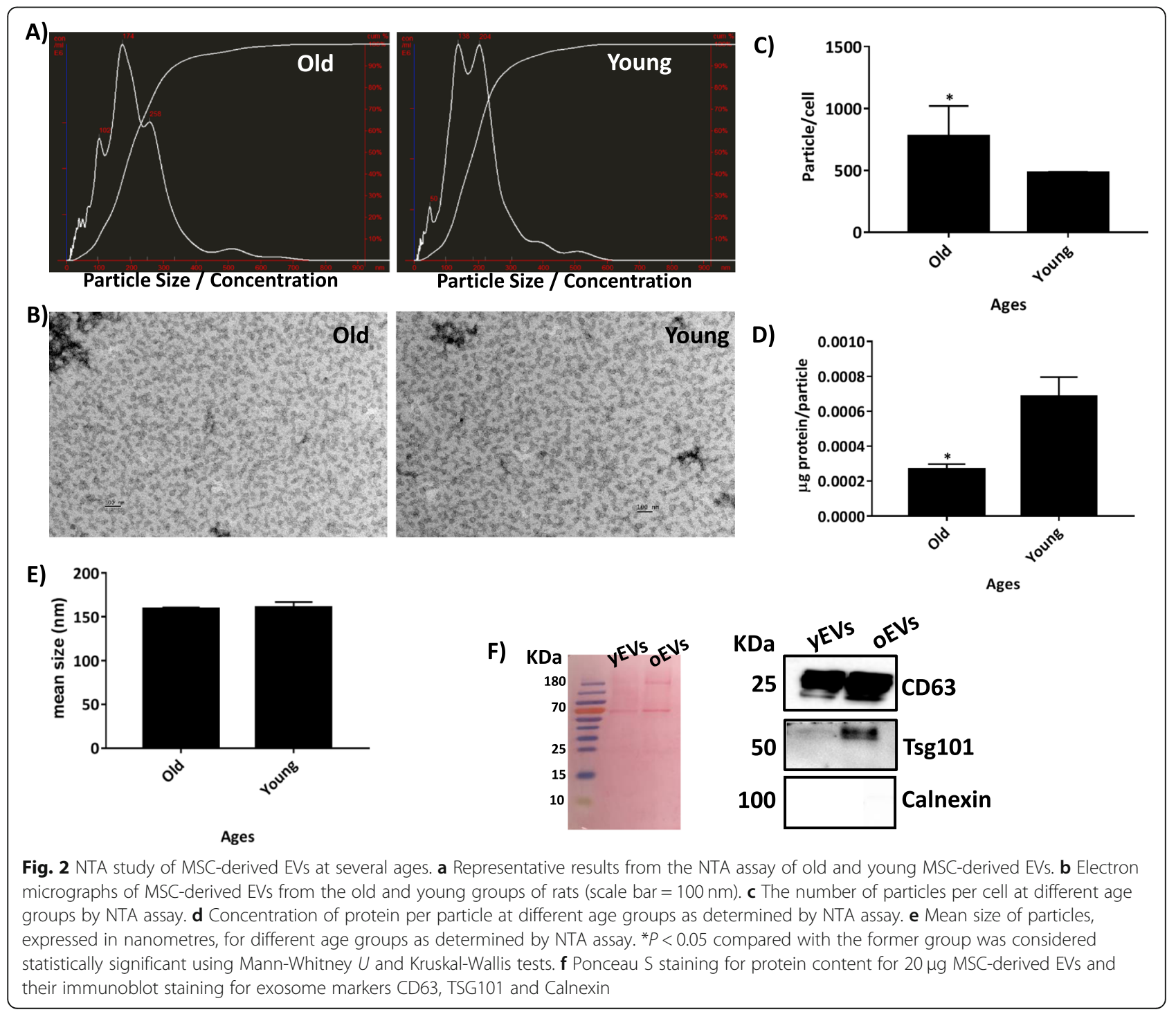




\section{Results}

Characterisation of both young and old populations of MSCs by flow cytometry revealed no statistically significant differences in levels of mesenchymal and haematopoietic markers (Fig. 1a). Cells positive for CD45 and CD34 represented less than $1 \%$ of cells positive for CD29 (70 $\pm 5 \%)$ and cells positive for CD90 (90 $\pm 5 \%)$, in all groups studied (Fig. 1b). There were no morphological differences between young and old MSCs (Fig. 1c), with both of them presenting as spindle-shaped.

Representative graphics obtained by nanoparticle tracking analysis (NTA) from old and young MSDderived EVs are shown in Fig. 2a. MSC-derived EVs were visualised by electronic microscopy as 70-80$\mathrm{nm}$-diameter vesicles in both old and young populations (Fig. 2b). The production of MSC-derived EVs increased with increasing donor age in a statistically significant way (52\%; Fig. 2c). By contrast, the protein to particle ratio of MSC-derived EVs decreased with increasing donor age in a statistically significant way (37.5\%; (Fig. 2d). The diameter of EVs as determined by NTA was $160 \pm 18 \mathrm{~nm}$ and was not statistically significantly different between the young and old groups (Fig. 2e). Western blotting analysis indicated that the MSC-derived EVs contained exosome-associated proteins CD63 and TSG101 but not calnexin (Fig. 2f).

Internalising of old MSC-derived EVs into young MSCs was observed after 2 days in culture, and there was an increase in EVs inside the MSCs with time
(Fig. 3a). A similar effect was observed by fluorescence microscopy in old MSCs co-cultured with young MSCderived EVs (Fig. 3b). Additional file 1: Figure S1 shows an old MSC with its cytoplasm full of young MSCderived EVs, at a magnification of $\times 100$.

Genes Nanog and Oct4, which are pluripotency markers in MSCs, showed statistically significant increased expression in old MSCs treated with young MSC-derived EVs at different times of incubation, compared to untreated cells $(P<0.05$; Fig. 4a). By contrast, young MSCs treated with old MSC-derived EVs showed statistically significant lower Nanog and Oct4 gene expression after 2 days of incubation $(P<0.05$; Fig. 4a). Vinculin, a senescence marker, had a statistically significant decrease in its expression at 6 days for old MSCs treated with young MSC-derived EVs $(P<0.05$; Fig. 4b), while it was increased statistically in young MSCs treated with old MSC-derived EVs $(P<0.05$; Fig. 4b).

Gene LMNA and its isoforms, as well as vinculin, which were senescence markers in MSCs, showed a statistically significant decrease in expression in old MSCs treated with young MSC-derived EVs at different times of incubation, compared to untreated cells $(P<0.05$; Fig. 5a). Young MSCs treated with old MSC-derived EVs also presented with statistically higher LMNA gene expression after 2, 3 and 6 days of incubation $(P<0.05$; Fig. 5a). Validation of levels of LMNA and its isoforms at the protein level indicated that old MSCs treated with young MSC-derived EVs had a decrease in the expression of isoforms lamin $\mathrm{A} / \mathrm{C}$ at 6 days (Fig. 5b), while

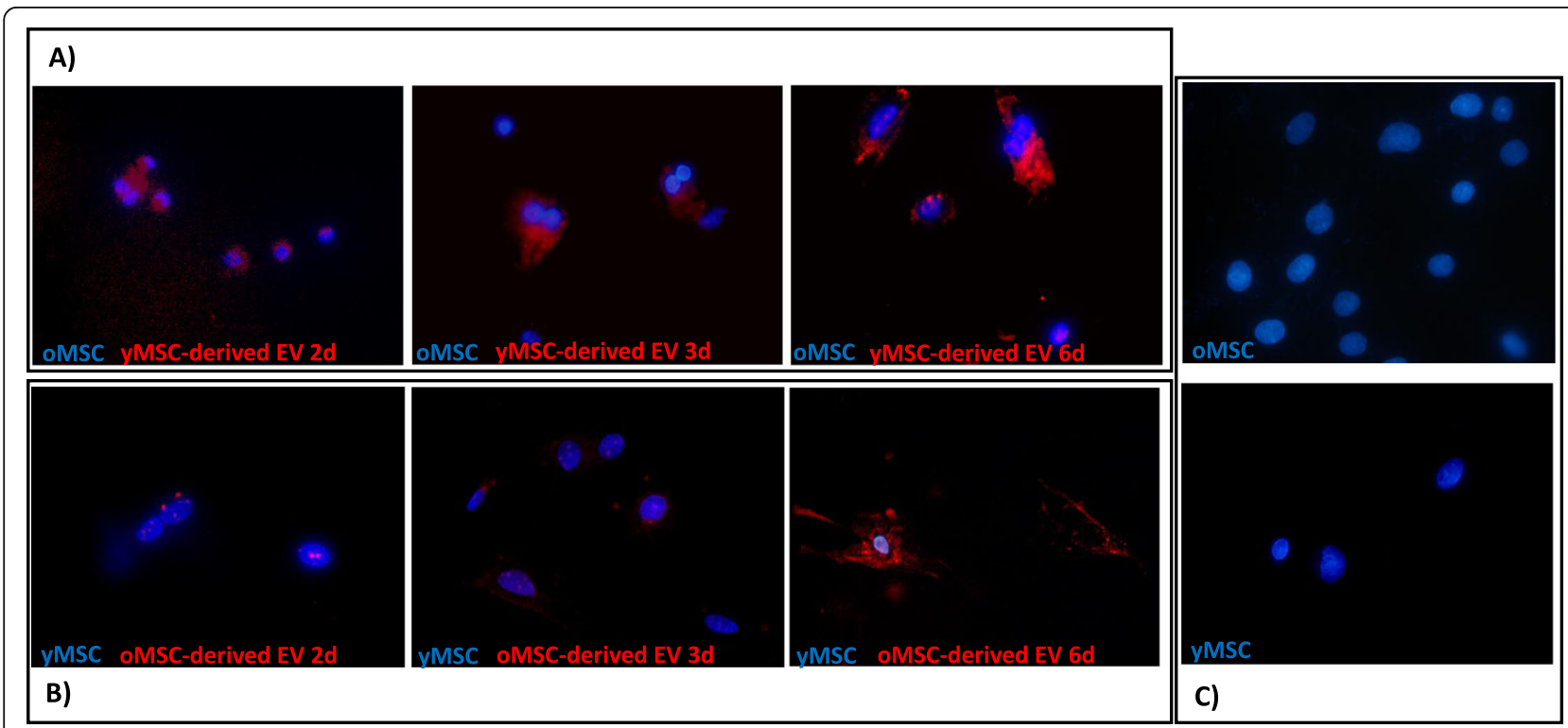

Fig. 3 Internalisation of MSC-derived EVs as observed by fluorescence microscopy. Images from fluorescence microscopy (magnification, $\times 40)$ of the nuclei of MSCs stained with 4',6-diamidino-2-phenylindole (DAPI) and MSC-derived EVs stained with Dil. a Old MSCs cultured with young MSC-derived EVs at 2, 3 and 6 days. b Young MSCs cultured with old MSC-derived EVs at 2, 3 and 6 days. c Control of young MSCs and old MSCs without added EVs. One representative experiment is shown. OMSC, MSCs from the old group; yMSC, MSCs from the young group; oEV, MSCderived EVs from the old group; yEV, MSC-derived EVs from the young group 


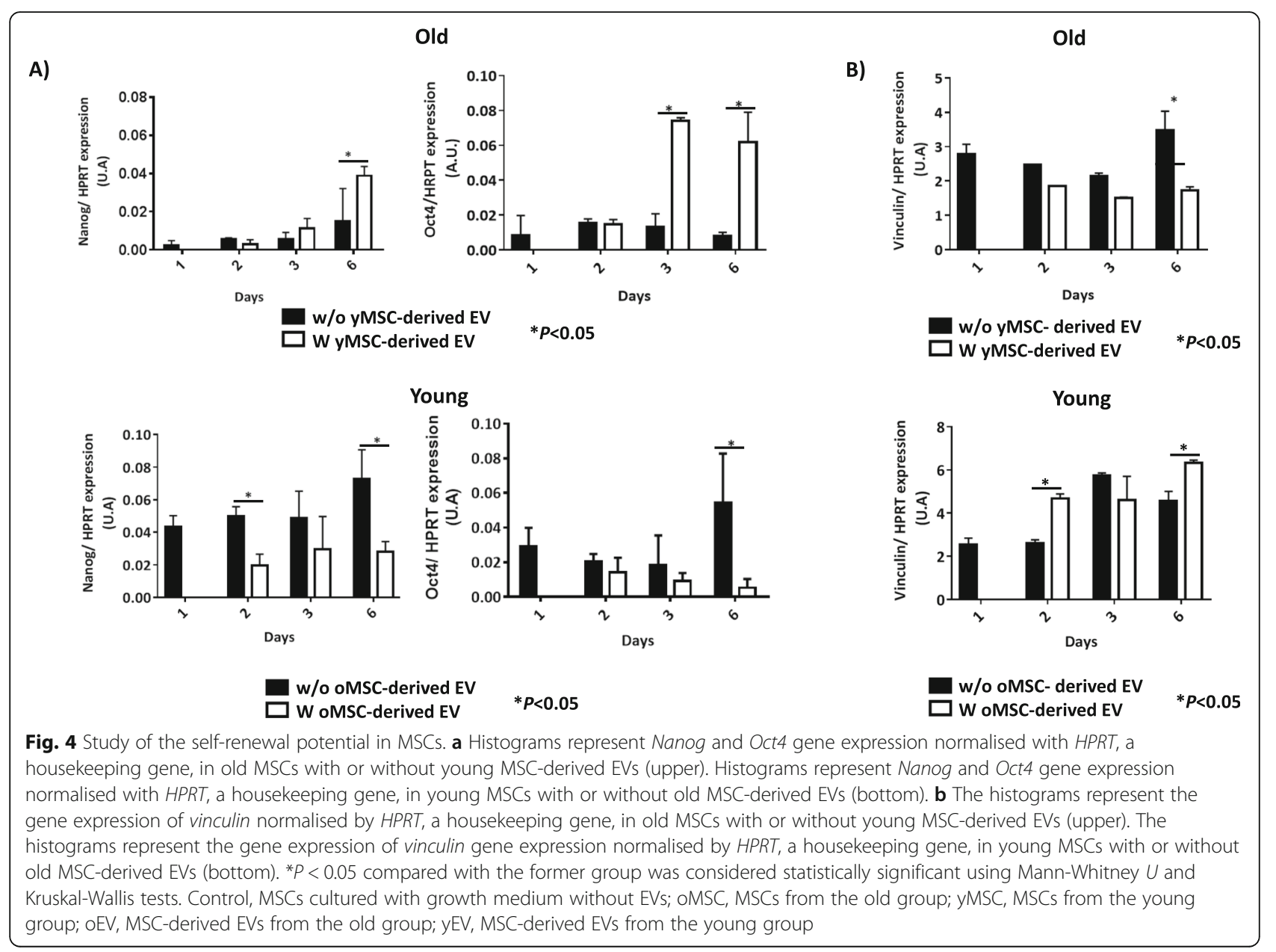

young MSCs treated with old MSC-derived EVs showed an increased expression at 6 days (Fig. 5b).

Study of the members of the mTOR pathway revealed statistically significant decreased expression of $m T O R$ $(P<0.05)$ in old MSCs treated with young MSC-derived EVs, after 2 and 6 days $(P<0.05$; Fig. 6a). Rictor and $A K T$ expression was also decreased; however, it was not statistically significant (Fig. 6a). By contrast, in young MSCs treated with old MSC-derived EVs, $m T O R$ and $A K T$ gene expression was significantly higher $(P<0.05)$ after 2, 3 and 6 days. Rictor revealed a statistically significant decrease in expression when young MSCs were treated with old MSC-derived EVs, after 2, 3 and 6 days $(P<0.05$; Fig. 6b). Western blot analysis indicated that levels of phosphor-mTOR and phosphor-AKT were decreased in old MSCs treated with young MSC-derived EVs, while Rictor was not detected (Fig. 6c). By contrast, western blot analysis indicated that phosphor-mTOR was increased in young MSCs treated with old MSCderived EVs. In addition, Rictor was detected in young MSCs treated with old MSC-derived EVs after 2 days in culture (Fig. 6d).
A statistically significant decrease in the miR-188 expression was obtained in young MSCs when treated with the inhibitor of miR-188, compared with mimic-miR188 used as a control to confirm transfection efficiency $\left(P<0.05\right.$; Fig. 7a). Young MSC-derived EVs- $188^{-/-}$were used to treat old MSCs, resulting in a statistically significant increase in the Rictor expression in this population, compared with old MSCs treated with only young MSCderived EVs (Fig. 7b). By contrast, the gene AKT showed decreased expression. These results were independently validated by western blot (Fig. 7c), where old MSCs treated with young MSC-derived $\mathrm{EVs}-188^{-/-}$produced an increased level of Rictor and a decreased level of phosphor-AKT. Young MSC-derived EVs- $188^{-/-}$produced a statistically significant decrease in the number of cells staining positively for SA- $\beta$-gal (Fig. $7 d$ ).

\section{Discussion}

The current study indicated that MSC-derived EVs might be involved in the senescence process, which could be influenced by Rictor through mTORC2. MSCs have been used therapeutically in regenerative medicine 
A)

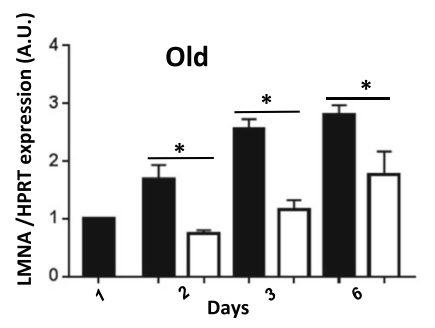

W/O yMSC-derived EV W yMSC-derived EV

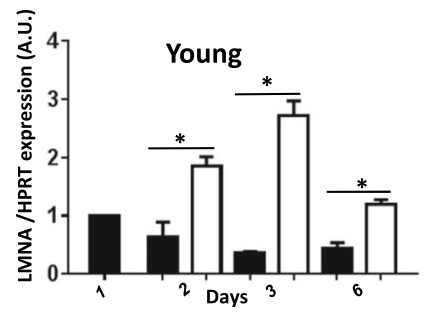

B)
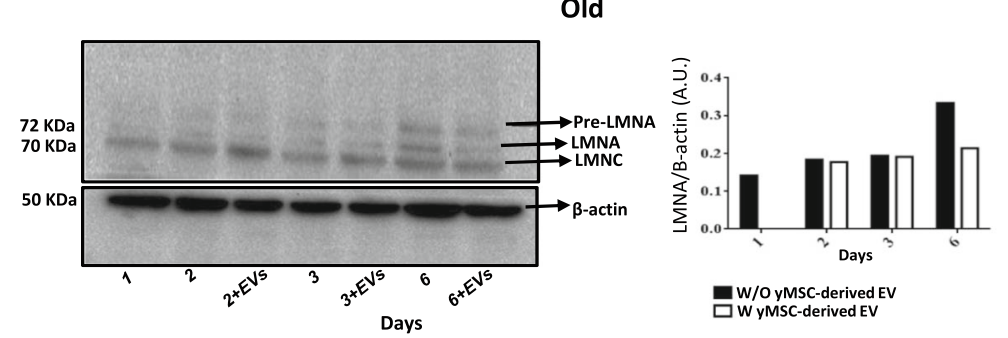

Young
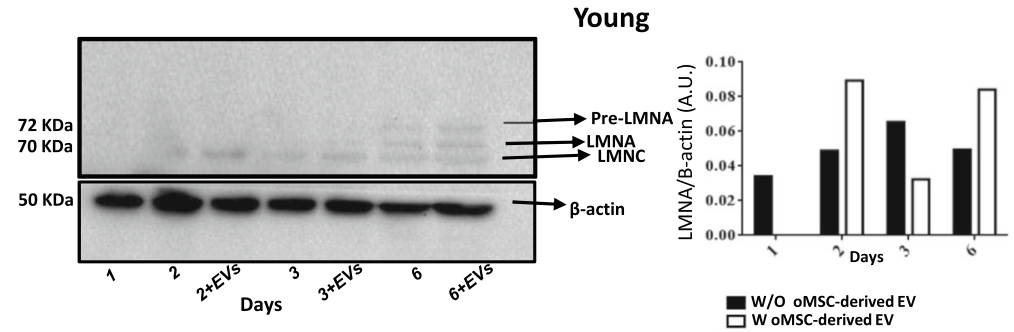

W/O oMSC-derived EV
$\square$ W oMSC-derived EV

Fig. 5 Study of senescence in MSCs. a The histograms represent the gene expression of LMNA normalised by HPRT, a housekeeping gene, in old and young MSCs with or without young or old MSC-derived EVs. ${ }^{*} P<0.05$ compared with the control was considered statistically significant using Mann-Whitney $U$ and Kruskal-Wallis tests. Control, MSCs cultured with growth medium without EVs; oMSC, MSCs from the old group; yMSC, MSCs from the young group; oEV, MSC-derived EVs from the old group; yEV, MSC-derived EVs from the young group. $\mathbf{b}$ Western blot analysis of pre-lamin A and their isoforms, lamin AVC, from old and young MSCs with or without old or young MSC-derived EVs at different times (2, 3 and 6 days) and LMNA densitometry analysis normalised with respect to $\beta$-actin, using Image Quant 5.2. The molecular weight of each protein is shown on the left. One representative experiment is shown. The gels have been run under the same experimental conditions

for their differentiation and secretory potential. Biancone et al. [2] have reported that MSCs can modulate gene expression by releasing extracellular vesicles to maintain physiological processes.

MSCs from two different age groups of rats were characterised by flow cytometry (Fig. 1), and our results were consistent with those of Jin et al. [10]. All the cells had the ability to adhere to the plastic culture dish, which was an inherent characteristic of MSCs, and the groups were able to differentiate towards several mesoderm lineages, results that were similar to a previously published report [11]. The size of EVs was determined by NTA, which calculated the size from the total concentration of vesicles in solution, following the technique used by Gercel-Taylor et al. [12] and validated by electron microscopy (Fig. 2b). We found an increase in the production of MSC-derived EVs from the old group of rats compared to the young group (Fig. 2c). A rational explanation for this fact could be as a compensation mechanism by old MSCs because of a decrease in their protein to particle ratio (Fig. 2d). These results were similar to those previously published by our group [4]. In addition, the MSC-derived EVs were positives for CD63 and Tsg101 but negative for calnexin, by western blot (Fig. 2f), meeting the requirements for their definition as EVs as reported by the International Society for Extracellular Vesicles (ISEV) [13].

There is an interesting study about the mechanisms of EV uptake by target cells, such as endocytosis mediated by clathrin or caveolin-dependent, macropinocytosis and phagocytosis [5], mechanisms involving cell surface membrane fusion with lipidic participation [6]. In addition, by fluorescence microscopy, this current study demonstrated the internalisation of MSC-derived EVs labelled with DiI, a process that was independent of the age of the donor (Fig. 3). These results were similar to other studies where dendritic cells [8] and melanoma cells [14] were used. In addition, some Dil label was observed in the extracellular region that could represent lipids from the membranes of MSC-derived EVs, as their lipid composition was abundant in complex phosphoryl derivatives of sphingosine and choline, which would support the plasma membrane renewing [15]. Young MSC-derived EVs labelled with DiI inside old MSCs is shown in Additional file 1: Figure S1.

The discovery of epigenetic mechanisms has placed the dynamic of global chromatin centrally to the following of pluripotency, and lineage progression of human embryonic stem cells because of the histone deacetylation is necessary to express Oct4 and Nanog in these cells [16]. Therefore, we performed genetic studies to evaluate the expression of those pluripotency markers, 


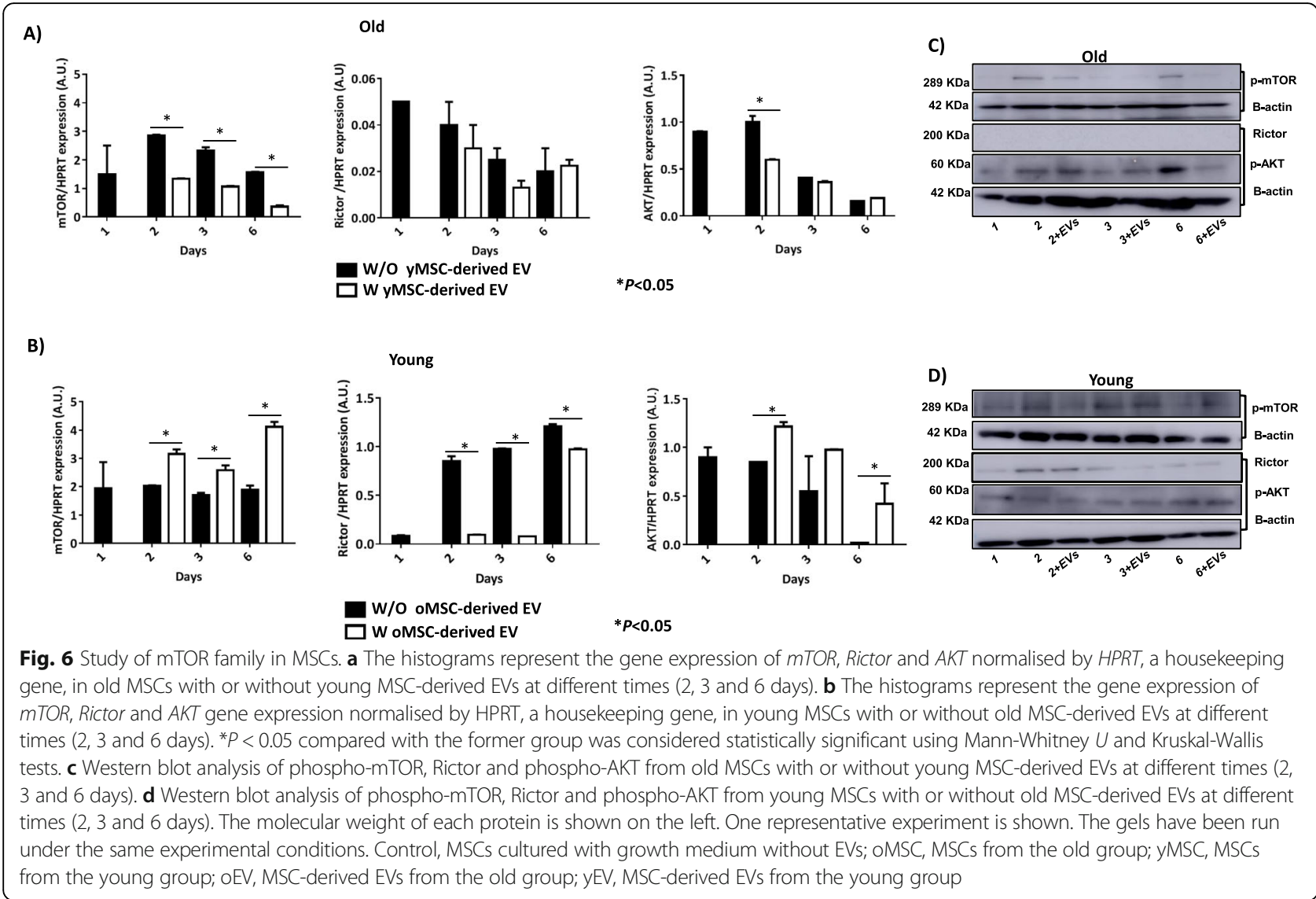

which are important transcription factors in controlling the expression of multiples genes associated with MSC pluripotency pathways. Reduced expression of these genes due to inactivation in in vitro culture under normoxia [11] results in reduced proliferation and pluripotency capacities [17]. It was observed that pluripotency expression markers changed when MSCs were incubated with MSC-derived EVs. Old MSCs showed increased expression of Nanog and Oct4 when they were cultured with young MSC-derived EV. By contrast, young MSCs cultured with old MSC-derived EV showed decreased expression of these transcription factors (Fig. 4a). As such, we confirmed that EV influenced the self-renewal capacity of MSCs at the genetic level, consistent with Jo et al. [18], who used a system of nanovesicles engineered from embryonic stem cells. Merino-González et al. [19] reported that the secretion of soluble factors and the release of extracellular vesicles, such as exosomes, could mediate cellular communication, inducing cell differentiation and self-renewal.

Vinculin gene expression was assessed in this current study because it was involved in contractility and cellular adhesion [20]. Vinculin is a well-characterised F-actin binding protein localised in focal adhesions as well as in cell-adherence junctions and undergoes changes in the expression both at the proteomic and genetic levels in relationship with age. A decrease in the expression of this gene was observed in old MSC cultured with young MSC-derived EVs, while an increase was found in young MSCs cultured with old MSC-derived EVs (Fig. 4b). Another ageing marker studied was $L M N A$, whose expression is increased in premature ageing conditions, as is prelamin A [21]. An increase in the expression of the three lamin isoforms was observed by western blot technique in young MSCs co-cultured with old MSC-derived EVs, while old MSCs cultured with young MSC-derived EVs showed decreased levels (Fig. 5a, b), with the highest levels being found after 6 days. All these results indicated that EVs contained factors which either stimulated or inhibited targets in the cells to which they were added, consistent with the findings of Fierabracci et al. [22]. As such, we evaluated the changes at the protein levels and confirmed the influence of MSC-derived EVs on senescence of MSCs in vitro. This discovery suggested that old MSC-derived EVs contained "age-promoting" factors [23], which may be responsible for the age-associated decline in stem cells, self-renewal and pluripotency, which are influenced by ageing. It was an important concept in the understanding of the ageing process and in developing EV-based therapies. 


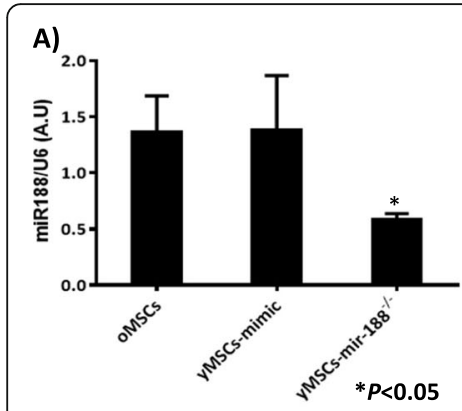

B)

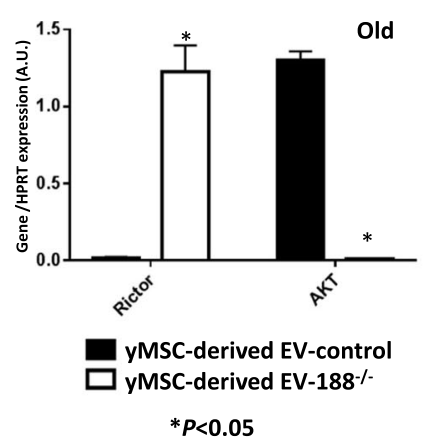

C)

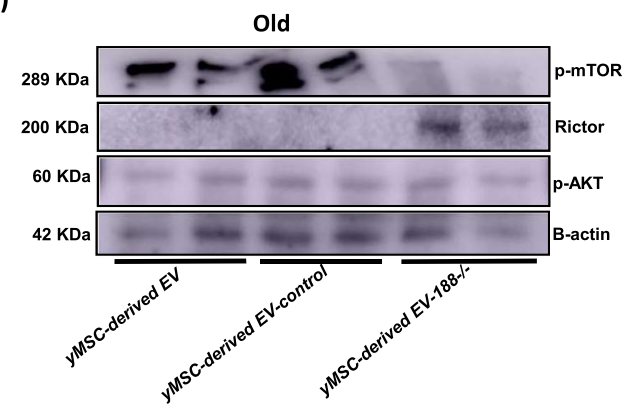

D)
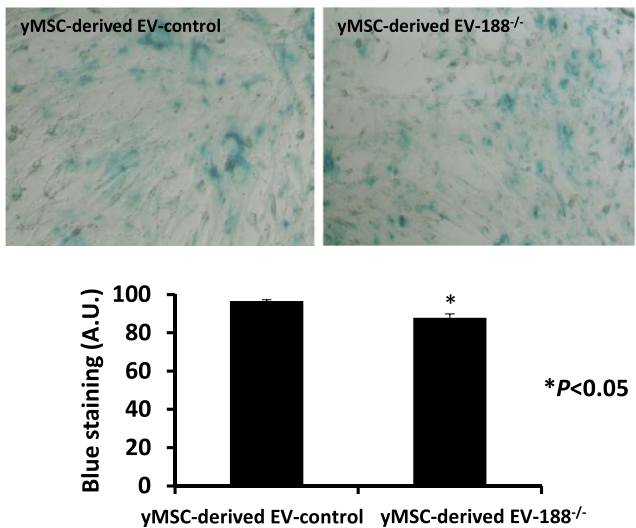

Fig. 7 Effect of inhibition of miR-188-3p in MSCs. a miR-188-3p expression in old MSCs and young MSCs transfected with mimic or miR-188-3p inhibitor, as determined by RT-qPCR analysis normalised with the expression of U6 small nuclear RNA. b Rictor and AKT gene expressions using RT-qPCR analysis normalised with the expression of HPRT in old MSCs treated with young MSC-derived EVs mimic used as a control and young MSC-derived EVs miR188 ${ }^{-/-} .{ }^{*} P<0.05$ compared with control was considered statistically significant using Mann-Whitney $U$ and Kruskal-Wallis tests. c Rictor and phosphor-AKT levels in old MSCS treated with young MSC-derived EV mimic used as control and young MSC-derived EVS miR1 $188^{-1}$, using western blot analysis normalised by the expression of $\beta$-actin. $\mathbf{d}$ Representative images (magnification, $\times 20$ ) of senescenceassociated beta-galactosidase (SA- $\beta$-gal) staining of old MSCs with young MSC-derived EVs and with young MSC-derived EVs modified with miR188-3p inhibitor added to the culture media, showing a higher number of negative cells in young MSC-derived EVs miR-188 ${ }^{-1-}$-treated cells in the densitometry analysis of the staining signal (bottom). Control, MSCs cultured with growth medium without EVs

Therefore, mTOR pathway represents a potential therapeutic target for improving defective, aged stem cells [4, 24]. mTOR showed decreased expression at the transcriptional (Fig. 6a) and post-transcriptional (phosphor-mTOR; Fig. 6c) levels when young MSCderived EVs were incubated with old MSCs. By contrast, we found the opposite results at the transcriptional (Fig. 6b) and post-transcriptional (Fig. 6d) levels when old MSC-derived EVs were incubated in young MSC cultures. Rictor did not show any variation in old MSCs treated with young MSC-derived EVs at the transcriptional level, and it was not detected at the post-transcriptional level (Fig. 6a, c). It could be able because the expression of Rictor in young MSCs is very low, and it is corroborated at post-transcriptional level (Fig. 6c). Young MSC-derived EVs produced a reduction in $A K T$ gene and AKT expressions (Fig. 6a, c). We observed that young MSC-derived EVs were more effective than old MSC-derived EVs in their influence on MSCs in vitro, as reported by several authors [25], except in the case of Rictor expression. The results obtained at the transcriptional and post-transcriptional levels showed a similar trend, although delayed in time, and this could be because unique features of MSCs are controlled by a dynamic interplay between extrinsic signalling pathways and regulation by epigenetic events not studied in this model. MiR-188-3p directly targets Rictor, as demonstrated by $\mathrm{Li}$ et al. [26] and Gharibi et al. [27], preventing the development of an age-related phenotype and maintaining MSC morphology of early passage cells, with high clonogenic frequency and enhanced proliferative capacity. Young MSCs showed knockdown on treatment with the antagonist of miR-188-3p (Fig. 7a) where miR-188-3p levels of the expression were significantly decreased, as well as its expression level in transfected young MSC-derived EVs (data not shown). These results were consistent with those published by Zou et al. [28] where miR-188-3p showed the same expression pattern in the cells as well as into 
their exosomes. Young MSC-derived EVs miR-188 ${ }^{-/-}$increased the expression of Rictor and decreased that of $A K T$ (Fig. $7 \mathrm{~b}$ ) at the transcriptional level, and phosphorylated forms of mTOR and AKT were confirmed by western blotting (Fig. 7c), accomplished with a significant reduction of SA- $\beta$-gal staining-positive cells in old MSCs treated with young MSC-derived EVs miR-188 ${ }^{-/-}$versus old MSCs treated only with regular young-derived EVs (Fig. 7d). We produced an inhibition of phosphor-AKT in old MSCs through the increase of Rictor expression, by inhibition of miR-188-3p, which induced rejuvenation of old MSCs, as evident by a reduction of their betagalactosidase staining. Therefore, bio-products from MSCs, such as extracellular vesicles, represent a promising new way of reprogramming resident cells in several diseases, such as acute and chronic kidney disease [29], or facilitating wound healing by promoting collagen synthesis and angiogenesis [30].

In conclusion, extracellular vesicles are a promising new approach to reprogramming cellular lines, modifying the expression of target families like mTOR involved in ageing, through their miR cargo.

\section{Supplementary information}

Supplementary information accompanies this paper at https://doi.org/10. 1186/s13287-019-1534-0.

Additional file 1: Figure S1. Young MSCs-derived EVs labelled with Dil inside old MSCs is shown.

\begin{abstract}
Abbreviations
DAPI: 4',6-Diamidino-2-phenylindole; Dil: 3-3'-Diethylthiacarbocyanineiodide; EVs: Extracellular vesicles; HPRT: Hypoxanthine-guanine

phosphoribosyltransferase; miRNA: MicroRNA; MSCs: Mesenchymal stem cells; MSC-derived EVs: Mesenchymal stem cell-derived extracellular vesicles; NTA: Nanoparticle tracking analysis; oEV: Mesenchymal stem cell-derived extracellular vesicles from the old group; oMSC: Mesenchymal stem cells from the old group; qRT-PCR: Real-time quantitative reverse transcription polymerase chain reaction; SA- $\beta$-gal: Senescence-associated betagalactosidase; yEV: Mesenchymal stem cell-derived extracellular vesicles from the young group; yMSC: Mesenchymal stem cells from the young group
\end{abstract}

\section{Acknowledgements}

We are very grateful to Noa Goyanes for the excellent technical help.

\section{Authors' contributions}

$J F-L$ and MM- $L$ carried out all the experiments. MJS-D realised the isolation of MSCs. OJA and FAJVDL realised the NTA study. JDT realised the important suggestions which improved the manuscript. MCA conceived the study, participated in its design and coordination, and drafted the manuscript. JF-L, MM-L, MJS-D, OJA, FAJVDL, JDT and MCA have given approval for the final version to be published.

\section{Funding}

JAF-L is the recipient of a postdoctoral fellowship funded by Consellería de Cultura, Educación e Ordenación Universitaria, Xunta de Galicia (Spain).

\section{Availability of data and materials}

All data generated during this study are included in this published article and its supplementary information files.

\section{Ethics approval and consent to participate}

All methods were carried out in accordance with the approved guidelines of the Spanish Law (32/2007). All experimental protocols were approved by the Animal Ethical Committee of Galicia.

\section{Consent for publication}

Not applicable

\section{Competing interests}

The authors declare that they have no competing interests.

\section{Author details}

${ }^{1}$ Grupo de Terapia Celular y Medicina Regenerativa, Instituto de Investigación Biomédica de A Coruña (INIBIC), Complexo Hospitalario Universitario de A Coruña (CHUAC), 15006-A Coruña, Spain. ²Departamento de Fisioterapia, Ciencias Biomédicas y Medicina, Facultad de Ciencias de la Salud, Universidad de A Coruña (UDC), As Xubias, 15006-A Coruña, Spain. ${ }^{3}$ Experimental Rheumatology, Department of Rheumatology, Radboud University Medical Center, Nijmegen 6525 GA, The Netherlands.

Received: 23 April 2019 Revised: 27 November 2019 Accepted: 18 December 2019 Published online: 03 January 2020

References

1. Karaoz E, Aksoy A, Ayhan S, Sariboyaci AE, Kaymaz F, Kasap M. Characterization of mesenchymal stem cells from rat bone marrow: ultrastructural properties, differentiation potential and immunophenotypic markers. Histochem Cell Biol. 2009;132(5):533-46. https://doi.org/10.1007/ s00418-009-0629-6 PubMed PMID: 19688349.

2. Biancone L, Bruno S, Deregibus MC, Tetta C, Camussi G. Therapeutic potential of mesenchymal stem cell-derived microvesicles. Nephrol Dial Transplant. 2012;27(8):3037-42. https://doi.org/10.1093/ndt/gfs168 PubMed PMID: 22851627.

3. Fafián-Labora J, Fernández-Pernas P, Fuentes I, De Toro J, Oreiro N, SangiaoAlvarellos $\mathrm{S}$, et al. Influence of age on rat bone-marrow mesenchymal stem cells potential. Sci Rep. 2015;5:16765. https://doi.org/10.1038/srep16765 PubMed PMID: 26581954; PubMed Central PMCID: PMCPMC4652164.

4. Fafián-Labora J, Lesende-Rodriguez I, Fernández-Pernas P, Sangiao-Alvarellos $\mathrm{S}$, Monserrat L, Arntz OJ, et al. Effect of age on pro-inflammatory miRNAs contained in mesenchymal stem cell-derived extracellular vesicles. Sci Rep. 2017;7:43923. https://doi.org/10.1038/srep43923 Epub 2017/03/06, PubMed PMID: 28262816

5. Feng D, Zhao WL, Ye YY, Bai XC, Liu RQ, Chang LF, et al. Cellular internalization of exosomes occurs through phagocytosis. Traffic. 2010;11(5): 675-87. https://doi.org/10.1111/j.1600-0854.2010.01041.x PubMed PMID: 20136776

6. Feng J, Li A, Deng J, Yang Y, Dang L, Ye Y, et al. miR-21 attenuates lipopolysaccharide-induced lipid accumulation and inflammatory response: potential role in cerebrovascular disease. Lipids Health Dis. 2014;13:27. https://doi.org/10.1186/1476-511X-13-27 PubMed PMID: 24502419; PubMed Central PMCID: PMCPMC3922422.

7. Debacq-Chainiaux F, Erusalimsky JD, Campisi J, Toussaint O. Protocols to detect senescence-associated beta-galactosidase (SA-betagal) activity, a biomarker of senescent cells in culture and in vivo. Nat Protoc. 2009;4(12): 1798-806. https://doi.org/10.1038/nprot.2009.191 PubMed PMID: 20010931.

8. Kharmate G, Hosseini-Beheshti E, Caradec J, Chin MY, Tomlinson Guns ES. Epidermal growth factor receptor in prostate cancer derived exosomes. PLoS One. 2016;11(5):e0154967. https://doi.org/10.1371/journal.pone. 0154967 Epub 2016/05/06. PubMed PMID: 27152724; PubMed Central PMCID: PMCPMC4859494.

9. Livak KJ, Schmittgen TD. Analysis of relative gene expression data using real-time quantitative $P C R$ and the $2-\Delta \Delta C T$ method. Methods. 2001;25(4): 402-8. https://doi.org/10.1006/meth.2001.1262 PubMed PMID: 11846609.

10. Jin HJ, Bae YK, Kim M, Kwon SJ, Jeon HB, Choi SJ, et al. Comparative analysis of human mesenchymal stem cells from bone marrow, adipose tissue, and umbilical cord blood as sources of cell therapy. Int J Mol Sci. 2013;14(9): 17986-8001. https://doi.org/10.3390/ijms140917986 PubMed PMID: 24005862: PubMed Central PMCID: PMCPMC3794764.

11. Kallas A, Pook M, Trei A, Maimets T. SOX2 is regulated differently from NANOG and OCT4 in human embryonic stem cells during early differentiation initiated with sodium butyrate. Stem Cells Int. 2014;2014: 
298163. https://doi.org/10.1155/2014/298163 Epub 2014/02/19. PubMed PMID: 24707296; PubMed Central PMCID: PMCPMC3951062.

12. Gercel-Taylor C, Atay S, Tullis RH, Kesimer M, Taylor DD. Nanoparticle analysis of circulating cell-derived vesicles in ovarian cancer patients. Anal Biochem. 2012;428(1):44-53. https://doi.org/10.1016/j.ab.2012.06.004 PubMed PMID: 22691960.

13. Witwer KW, Van Balkom BWM, Bruno S, Choo A, Dominici M, Gimona M, et al. Defining mesenchymal stromal cell (MSC)-derived small extracellular vesicles for therapeutic applications. J Extracell Vesicles. 2019;8(1):1609206. https://doi.org/10.1080/20013078.2019.1609206 Epub 2019/04/29. PubMed PMID: 31069028; PubMed Central PMCID: PMCPMC6493293.

14. Parolini I, Federici C, Raggi C, Lugini L, Palleschi S, De Milito A, et al. Microenvironmental $\mathrm{pH}$ is a key factor for exosome traffic in tumor cells. J Biol Chem. 2009;284(49):34211-22. https://doi.org/10.1074/jbc.M109.041152 Epub 2009/09/30. PubMed PMID: 19801663; PubMed Central PMCID: PMCPMC2797191.

15. Morelli AE, Larregina AT, Shufesky WJ, Sullivan ML, Stolz DB, Papworth GD, et al. Endocytosis, intracellular sorting, and processing of exosomes by dendritic cells. Blood. 2004;104(10):3257-66. https://doi.org/10.1182/blood2004-03-0824 Epub 2004/07/29. PubMed PMID: 15284116.

16. Parsons XH. Embedding the future of regenerative medicine into the open epigenomic landscape of pluripotent human embryonic stem cells. Annu Res Rev Biol. 2013;3(4):323-49 PubMed PMID: 25309947; PubMed Central PMCID: PMCPMC4190676.

17. Muñoz Descalzo S, Rué P, Garcia-Ojalvo J, Martinez AA. Correlations between the levels of Oct4 and Nanog as a signature for naive pluripotency in mouse embryonic stem cells. Stem Cells. 2012;30(12):2683-91. https://doi. org/10.1002/stem.1230 PubMed PMID: 22969005.

18. Jo W, Jeong D, Kim J, Park J. Self-renewal of bone marrow stem cells by nanovesicles engineered from embryonic stem cells. Adv Healthc Mater. 2016;5(24):3148-56. https://doi.org/10.1002/adhm.201600810 Epub 2016/11/ 10. PubMed PMID: 27860451.

19. Merino-González C, Zuñiga FA, Escudero C, Ormazabal V, Reyes C, NovaLamperti $\mathrm{E}$, et al. Mesenchymal stem cell-derived extracellular vesicles promote angiogenesis: potencial clinical application. Front Physiol. 2016;7: 24. https://doi.org/10.3389/fphys.2016.00024 Epub 2016/02/09. PubMed PMID: 26903875; PubMed Central PMCID: PMCPMC4746282.

20. Goldmann WH, Auernheimer V, Thievessen I, Fabry B. Vinculin, cell mechanics and tumour cell invasion. Cell Biol Int. 2013;37(5):397-405. https://doi.org/10.1002/cbin.10064 Epub 2013/03/13, PubMed PMID: 23494917.

21. Mateos J, Fafián-Labora J, Morente-López M, Lesende-Rodriguez I, Monserrat $L$, Ódena MA, et al. Next-generation sequencing and quantitative proteomics of Hutchinson-Gilford progeria syndrome-derived cells point to a role of nucleotide metabolism in premature aging. PLoS One. 2018;13(10): e0205878. https://doi.org/10.1371/journal.pone.0205878 Epub 2018/10/31, PubMed PMID: 30379953

22. Fierabracci $A$, Del Fattore $A$, Muraca $M$. The immunoregulatory activity of mesenchymal stem cells: 'state of art' and 'future avenues'. Curr Med Chem. 2016;23(27):3014-24 PubMed PMID: 27356536.

23. Ahlqvist KJ, Suomalainen A, Hämäläinen RH. Stem cells, mitochondria and aging. Biochim Biophys Acta. 2015;1847(11):1380-6. https://doi.org/10.1016/ j.bbabio.2015.05.014 Epub 2015/05/23, PubMed PMID: 26014347.

24. Takayama K, Kawakami Y, Lavasani M, Mu X, Cummins JH, Yurube T, et al. mTOR signaling plays a critical role in the defects observed in musclederived stem/progenitor cells isolated from a murine model of accelerated aging. J Orthop Res. 2016. https://doi.org/10.1002/jor.23409 Epub 2016/08/ 30, PubMed PMID: 27572850

25. Lei Q, Liu T, Gao F, Xie H, Sun L, Zhao A, et al. Microvesicles as potential biomarkers for the identification of senescence in human mesenchymal stem cells. Theranostics. 2017;7(10):2673-89. https://doi.org/10.7150/thno. 18915 Epub 2017/07/06, PubMed PMID: 28819455; PubMed Central PMCID: PMCPMC5558561.

26. Li CJ, Cheng P, Liang MK, Chen YS, Lu Q, Wang JY, et al. MicroRNA-188 regulates age-related switch between osteoblast and adipocyte differentiation. J Clin Invest. 2015;125(4):1509-22. https://doi.org/10.1172/ JCl77716 Epub 2015/03/09, PubMed PMID: 25751060; PubMed Central PMCID: PMCPMC4396470.

27. Gharibi B, Farzadi S, Ghuman M, Hughes FJ. Inhibition of Akt/mTOR attenuates age-related changes in mesenchymal stem cells. Stem Cells. 2014. https://doi.org/10.1002/stem.1709 PubMed PMID: 24659476.
28. Zou X, Li M, Huang Z, Zhou X, Liu Q, Xia T, et al. Circulating miR-532-502 cluster derived from chromosome $\mathrm{X}$ as biomarkers for diagnosis of breast cancer. Gene. 2019;144104. https://doi.org/10.1016/j.gene.2019.144104 Epub 2019/09/04, PubMed PMID: 31493506.

29. Nassar W, El-Ansary M, Sabry D, Mostafa MA, Fayad T, Kotb E, et al. Umbilical cord mesenchymal stem cells derived extracellular vesicles can safely ameliorate the progression of chronic kidney diseases. Biomater Res. 2016; 20:21. https://doi.org/10.1186/s40824-016-0068-0 Epub 2016/08/05, PubMed PMID: 27499886; PubMed Central PMCID: PMCPMC4974791.

30. Feng Y, Sanders AJ, Morgan LD, Harding KG, Jiang WG. Potential roles of suppressor of cytokine signaling in wound healing. Regen Med. 2016;11(2): 193-209. https://doi.org/10.2217/rme.16.4. PubMed PMID: 26877242.

\section{Publisher's Note}

Springer Nature remains neutral with regard to jurisdictional claims in published maps and institutional affiliations.
Ready to submit your research? Choose BMC and benefit from:

- fast, convenient online submission

- thorough peer review by experienced researchers in your field

- rapid publication on acceptance

- support for research data, including large and complex data types

- gold Open Access which fosters wider collaboration and increased citations

- maximum visibility for your research: over $100 \mathrm{M}$ website views per year

At BMC, research is always in progress.

Learn more biomedcentral.com/submissions 